Article

\title{
Identification of Buried Objects in GPR Using Amplitude Modulated Signals Extracted from Multiresolution Monogenic Signal Analysis
}

\author{
Lihong Qiao ${ }^{1,2}$, Yao Qin ${ }^{1,2, *}$, Xiaozhen Ren ${ }^{1,2}$ and Qifu Wang ${ }^{3}$ \\ Received: 31 July 2015; Accepted: 25 November 2015; Published: 4 December 2015 \\ Academic Editor: Assefa M. Melesse \\ 1 College of Information Science and Engineering, Henan University of Technology, Zhengzhou 450001, China; \\ lhqiao@haut.edu.cn (L.Q.); rxz235@163.com (X.Z.) \\ 2 Key Laboratory of Grain Information Processing and Control, Henan University of Technology, \\ Ministry of Education, Zhengzhou 450001, China \\ 3 Henan Academy of Science, Applied Physics Institute Co., Ltd, Zhengzhou 450001, China; \\ ewangqifu@hotmail.com \\ * Correspondence: eqinyao@163.com; Tel.: +86-27-6775-6529
}

\begin{abstract}
It is necessary to detect the target reflections in ground penetrating radar (GPR) images, so that surface metal targets can be identified successfully. In order to accurately locate buried metal objects, a novel method called the Multiresolution Monogenic Signal Analysis (MMSA) system is applied in ground penetrating radar (GPR) images. This process includes four steps. First the image is decomposed by the MMSA to extract the amplitude component of the B-scan image. The amplitude component enhances the target reflection and suppresses the direct wave and reflective wave to a large extent. Then we use the region of interest extraction method to locate the genuine target reflections from spurious reflections by calculating the normalized variance of the amplitude component. To find the apexes of the targets, a Hough transform is used in the restricted area. Finally, we estimate the horizontal and vertical position of the target. In terms of buried object detection, the proposed system exhibits promising performance, as shown in the experimental results.
\end{abstract}

Keywords: reflection hyperbola; GPR; multiresolution monogenic signal analysis; amplitude component; Hough transform

\section{Introduction}

As a geophysical method, ground penetrating radar (GPR) is used in the detection of the targets in near the ground surface areas for locating underground objects such as land mines and pipes. We focus on the metal point detection in this paper. All hyperbolas have to be located and each single radargram has to be analyzed, for the purpose of extracting the exact position of the targets.

There are some researches on buried object detection. To identify hyperbolas, Delbo et al. [1] propose a procedure based on wavelets to reduce noise and then a fuzzy cluster approach to detect objects. Artificial neural networks are used in [2]. A pattern recognition process is used to locate underground objects using a pattern recognition process [3,4]. Real-time interpretation of GPR images is proposed in [5].

A model called Multiresolution Monogenic Signal Analysis (MMSA) has been developed by Unser [6]. Multiple components such as amplitude components and phase are obtained using monogenic signal analysis, which has a proper multiresolution presentation. The wavelet-based amplitude components suppress noise and enhance targets in GPR images. Dong et al. [7] propose synthetic aperture radar (SAR) target classification method based on Riemannian geometry. Combined 
with a covariance matrix, the monogenic components are used in SAR image classification. Besides, sparse representation of the monogenic signal of SAR images is proposed in [8].

This paper aims to present a novel method for solving the object detection issues. The method includes preprocessing of the data using an MMSA to extract the amplitude component. After the extraction, background clutter is removed to a large extent while keeping the target signal, which aims to get rid of the false targets and locate the targets accurately. To limit the scope of reflection hyperbolas to certain areas, we use the ROI extraction method in the GPR data, which decreases the computational complexity of hyperbola fitting. Then, the Hough transform is used for hyperbola fitting. Finally, we estimate the horizontal and vertical position of the target. Experiments on GPR images come from the finite-difference time-domain (FDTD) method and realistic situations.

The paper is organized as follows: the reflection model of a buried object is introduced in Section 2. The detection algorithm based on MMSA, extraction of ROI and Hough transform application is introduced in Section 3. The experimental results are illustrated in Section 4. Finally, conclusions are presented in Section 5.

\section{Reflection Model of a Buried Object}

A radar system contains a transmitting and receiving antenna pair. First, we review the reflection model of a buried metal point target. When the ground-coupled antenna scans an object linearly, the antenna position $x$ and corresponding echo time delay $t$ approximately satisfy a three-parameter hyperbola equation:

$$
\frac{t^{2}}{t_{0}^{2}}-\frac{4\left(x-x_{0}\right)^{2}}{v^{2} t_{0}^{2}}=1
$$

where $v$ is the velocity of wave propagation underground, assumed to be constant in a small region, $x_{0}$ is the horizontal position of the object, and $t_{0}$ is the time delay for the echo. The apex $\left(x_{0}, t_{0}\right)$ indicates the object position of the vertex. The velocity $v$ and the position $\left(x_{0}, t_{0}\right)$ of the vertex determine the shape of the reflection hyperbola. A simple reflection model for GPR is shown in Figure 1.

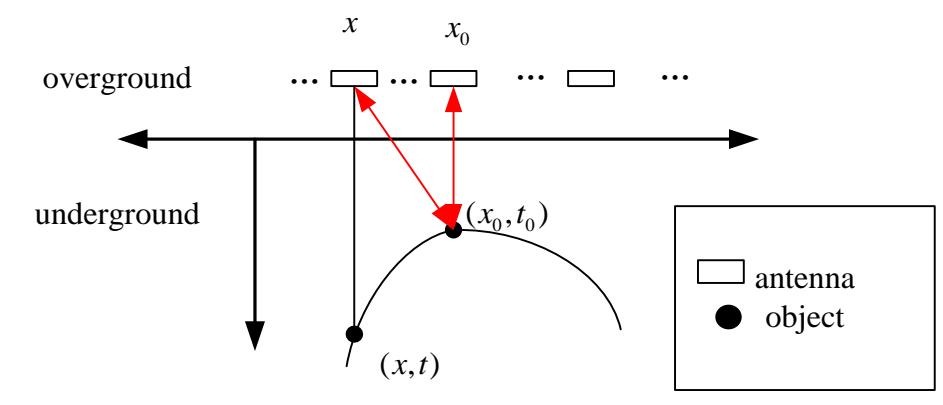

Figure 1. A simple reflection model for GPR. The antenna position $x$ and the echo time delay $t$ approximately satisfy the hyperbolic Equation (1).

\section{Detection Algorithm}

The object detection method consists of four steps:

1. Extract the amplitude components using the MMSA method;

2. Extract the Region of Interest to narrow down the region to certain areas;

3. Locate hyperbolic patterns via a Hough transformation;

4. Estimate the horizontal and vertical position of the target.

The flowchart is shown in Figure 2. 


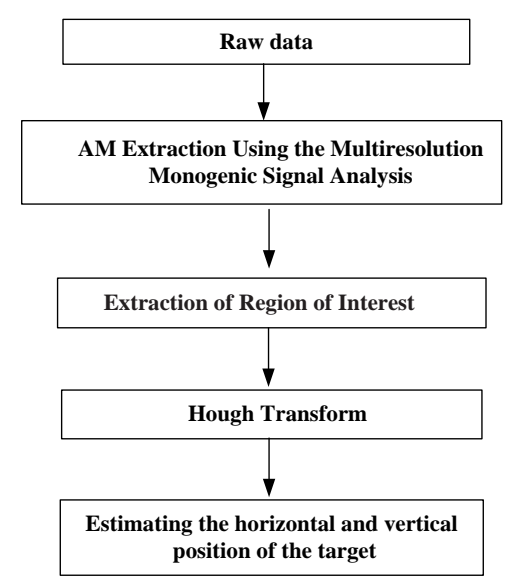

Figure 2. The flowchart of the proposed strategy.

In detail, the GPR image is first decomposed by the monogenic wavelet transform to extract the amplitude component. The amplitude component enhances the target reflection and suppresses the direct wave and reflective wave to a large extent. The phase information decomposed by the monogenic wavelet transform contains too much detailed texture information of all waves and it is hard to locate the target. The method can not only intensify the target, but also eliminate the undesired presence of the ground surface echo. Then we use the ROI extraction method to restrict the region to a small range. The extraction is based on the normalized variance bigger than a fixed number. The computational cost of the Hough transform is significantly reduced. Then the Hough transformation is used to detect hyperbolic patterns.

\subsection{Preprocessing Based on the Multiresolution Monogenic Signal Analysis Structure}

The MMSA method uses the Riesz-Laplace wavelet transforms. Three wavelet components-amplitude components, phase components and instantaneous frequency components-are extracted. These parameters have a very clear definition in radio frequency theory and they can be extended to two dimensional signals. Using the amplitude component, background clutter is removed to large extent while keeping the target signal.

In this section we first review the monogenic signal. Then, we introduce the Riesz-Laplace wavelet transforms to implement the MMSA method and get the modulation characteristics, which include amplitude, phase and frequency.

Three-components of monogenic signal $f(\chi)$ are defined in [9] as:

$$
\begin{aligned}
& f_{m}(\chi) \\
& =(f(\chi), \operatorname{Re}(\Re f(\chi)), \operatorname{Im}(\Re(f(\chi)))) \\
& =\left(f, f_{1}, f_{2}\right)
\end{aligned}
$$

where $f(\chi)$ is a two dimensional signal, $\chi=(x, y)$, and $\Re$ is the Riesz transform of $f(\chi)$.

Amplitude component is defined by:

$$
A(x)=\left\|f_{m}(x)\right\|=\sqrt{f^{2}+f_{1}^{2}+f_{2}^{2}},
$$

The orientation $\theta$ and phase $\xi$ are given by:

$$
f=A \cos \xi, f_{1}=A \sin \xi \cos \theta, f_{2}=A \sin \xi \sin \theta
$$


In particular, the amplitude components and phase components are recovered in the direction $\mathbf{u}=(\cos \theta, \sin \theta)=\left(f_{1} / r, f_{2} / r\right)$, which can be extended to other directions.

Then we show how to realize a monogenic representation of wavelet coefficient. On each bandpass-filtered signal:

$$
\left(f * \psi_{i}\right)(x)
$$

where $\psi_{i}(x)=|\operatorname{det}(D)|^{i / 2} \psi\left(D^{i} x\right)$ is the normalized and spine wavelet.

Due to the symmetry characteristics, the coefficients of the signal are defined as:

$$
\begin{gathered}
\omega_{i}[\mathbf{k}]=\left\langle f, \psi_{i, \mathbf{k}}\right\rangle=\left.\left(\psi_{i} * f\right)(x)\right|_{x=D^{-(i+1)} \mathbf{k}} \\
r_{1, i}[\mathbf{k}]+j r_{2, i}[\mathbf{k}]=\left\langle f, \Re * \psi_{i, \mathbf{k}}\right\rangle=\left.\Re\left\langle f * \psi_{i, \mathbf{k}}\right\rangle\right|_{x=D^{-(i+1)} \mathbf{k}}
\end{gathered}
$$

where Riesz coefficient's real component is $r_{1, i}[\mathbf{k}]$ and the imaginary component is $r_{2, i}[\mathbf{k}] . k$ is the space index and $i$ is the scale index.

Based on previous knowledge, the MMSA is applied to extract the amplitude components in the wavelet-domain. The tensor method is used to estimate the local orientation. The corresponding directional Hilbert component is given by:

$$
q_{i}[\mathbf{k}]=r_{1, i}[\mathbf{k}] \cos \theta+r_{2, i}[\mathbf{k}] \sin \theta
$$

Then the analytic representation is:

$$
\omega_{i}[\mathbf{k}]+q_{i}[\mathbf{k}] j=A_{i}[\mathbf{k}] \cdot e^{j \xi_{i}[\mathbf{k}]}
$$

Then the modulation components are defined by:

$$
\begin{gathered}
A_{i}[\mathbf{k}]=\sqrt{\omega_{i}[\mathbf{k}]^{2}+q_{i}[\mathbf{k}]^{2}} \\
\xi_{i}[\mathbf{k}]=\arctan \left(\frac{q_{i}[\mathbf{k}]}{\omega_{i}[\mathbf{k}]}\right)
\end{gathered}
$$

We use MMSA to define the amplitude component. The frequency component contains too much noise of the reflective wave and it is not so convenient to use this component. In monogenic representation, the local amplitude represents the local energetic information, whereas the phase depicts the local structural information. Thus, the monogenic signal fulfills the invariant property with respect to energy and orientation.

Figure 3 illustrates the ability of the MMSA method to extract the amplitude component. With a fixed Gaussian window, we use a tensor-based method to estimate the orientation. Figure 3a is a GPR experiment data profile. It contains recorded point data with a mean frequency of $300 \mathrm{MHz}$ and a time window of $100 \mathrm{~ns}$. The horizontal and vertical coordinates are the trace and sample points, respectively. The number of traces is 540 and the number of sampling points for each trace is 512. Figure $3 \mathrm{~b}$ illustrates the three amplitude component image extraction result under three level monogenic signal analysis. Three amplitude components are depicted in one picture. As compared with Figure 3a, the ground surface has obvious direct waves, and the first amplitude component suppresses the direct wave and reflective wave to a large extent. This representation not only enhances the target reflection, but also eliminates the redundant echo. Figure $3 \mathrm{c}$ shows the enlarged second amplitude-component. The amplitude component makes the target signals obvious. 


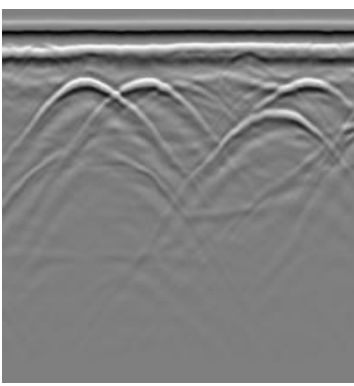

(a)

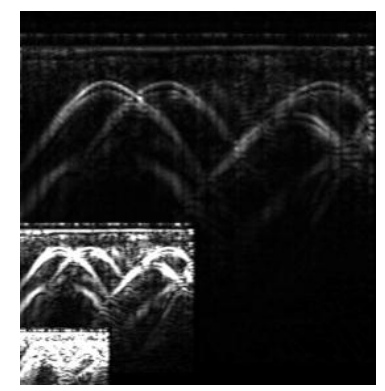

(b)

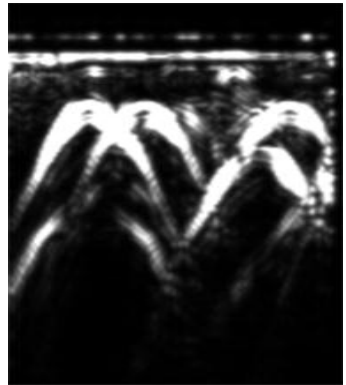

(c)

Figure 3. Example of amplitude components extracted by the MMSA method. (a) Input image; (b) Wavelet-domain amplitude estimation. Three amplitude components under three level monogenic wavelet analysis. The amplitude components eliminate the direct and surface-wave noise; (c) The enlarged second amplitude component.

\subsection{Extraction the Region of Interest}

Cheng proposed the Region of Interest (ROI) extraction method [10]. Based on the analysis of GPR A-Scan signal's statistical features, a windowed statistical method is used to extract the ROI from a large amount of data.

In detail, the variance of the B-scan data is calculated. Then the normalized variance of the data is calculated. We note that the variance is significantly larger in the target area. The ROI is extracted based on the normalized variance being bigger than a fixed number. The first amplitude component extracted by the MMSA is shown in Figure 4a. Then the normalized variance of amplitude component is illustrated in Figure $4 \mathrm{~b}$. The extraction of ROI of the amplitude is based on the normalized variance with a lower threshold. The extraction result is shown in Figure 4c. This method gets rid of the false targets and locates the targets accurately and it is especially used for the targets at almost the same depth.

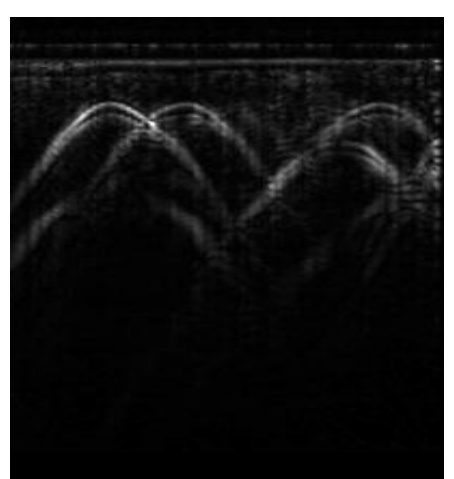

(a)

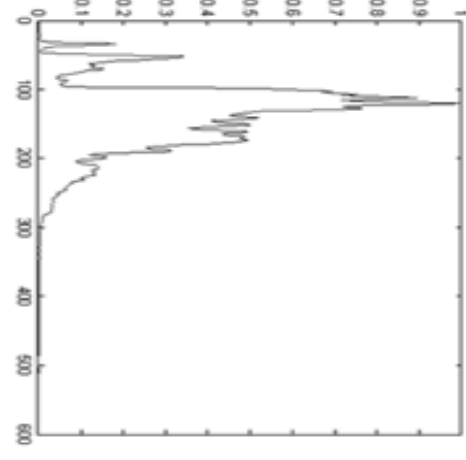

(b)

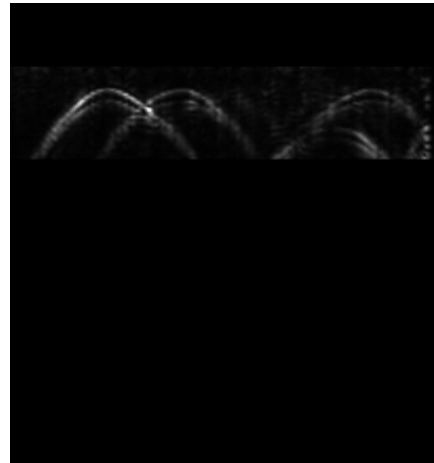

(c)

Figure 4. Example of the extraction of Region of Interest. (a) The first amplitude modulation component extracted by using the MMSA; (b) The normalized variance of amplitude component; (c) The extraction of ROI of the amplitude based on the normalized variance with a lower threshold.

\subsection{Hough Transform}

We briefly introduce the Hough transform as follows. We rearrange Equation (1):

$$
t_{0}=\sqrt{t^{2}-\frac{4}{v^{2}}\left(x-x_{0}\right)^{2}}
$$


where $(x, t)$ represents the reflection hyperbola's coordinates and $x_{0}, t_{0}$, and $v$ are the parameters. We first limit the image area by the ROI extraction algorithm, so the computational time decreases dramatically. The Hough transform used in our paper was shown in [11].

In our method, ROI limits the area before the Hough transform. In order to reduce noise and artifacts, the image in marked regions is smoothed with Gaussian filters. Then by a canny edge detector, this image is converted into a binary image. Then using the Hough transform, the position of the target is determined. In detail we define the number of accumulator-cells for $x_{0}$ and $t_{0}$ using the edge pixels. The propagation velocity is an important parameter to obtain the best fitting. It varies for different soil characteristics especially for large range of areas. The velocity $v$ in the Hough transform varies in a given interval assuming the range is not so big, which can be set by the user. Then we transform every edge pixel $(x, t)$ and every discrete value of $v$ into the Hough plane with Equation (1). Finally, we draw the location result in the original image (see Figure 5). Firstly, using the Gabor filter, the image in the extracted region is smoothed. By a canny detector, the image becomes a binary image (shown in the middle of Figure 5). Then we use Hough transform in the ROI region. The calculated hyperbolas are drawn in red color.

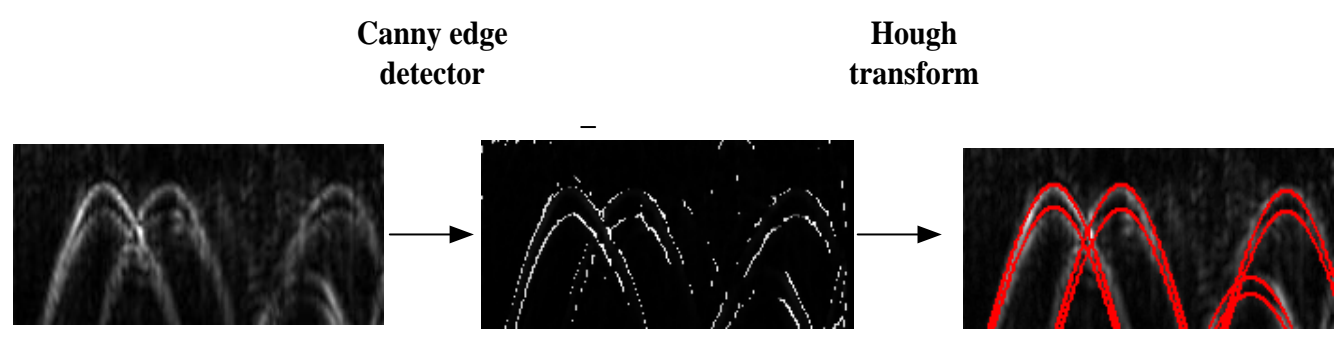

Figure 5. The result of the Hough transform. The image is in the ROI region (on the left). Then the canny edge detects result is shown. Then the Hough transform result. The hyperbola is drawn in red color.

\section{Results}

We use GPR numerical simulation images and experimental images to assess the target detection. The electromagnetic simulator "GprMax" is used to generate simulation images using the finite difference time domain (FDTD) method. RIS radar is applied to collect the experimental data.

\subsection{Simulation Results}

The whole simulation process is shown in Figure 6. Figure 6a illustrates a scenario of metal blocks in three distinct steps. This is a simulated image. The width and depth are $2.8 \mathrm{~m}$ and $3 \mathrm{~m}$, respectively. The background medium is dry sand and three perfect conductor metal cylinders with $0.01 \mathrm{~m}$ diameter are buried at the same depth of $0.45 \mathrm{~m}$. The interval of the three metal targets is $1 \mathrm{~m}$. In the simulation model, the grid in the $x$ and $y$ direction is $0.01 \mathrm{~m}$ and detection step is $0.02 \mathrm{~m}$ in the $x$ direction. All the boundaries are Perfectly Matched Layers (PMLs). The parameter of the current definition is a Hertzian dipole with unit amplitude and center frequency of a Ricker wavelet of $600 \mathrm{MHz}$, and the time window for each trace is $25 \mathrm{~ns}$. There are 141 traces in total and 1060 samples. The interval between samples is $2.3587 \times 10^{-11} \mathrm{~s}$. The actual electromagnetic wave velocity is $14.8 \mathrm{~cm} / \mathrm{ns}$. The related GPR image is shown in Figure $6 \mathrm{~b}$ which is generated by the "GprMax" simulator. The extraction result of the first amplitude component of the MMSA step is shown in Figure 6c, where the reflection signals and the noise can be suppressed and the target reflection can be enhanced after the extraction of the amplitude component. Then the ROI extraction method is used to get rid of the false targets and locate the targets accurately. The result is illustrated in Figure $6 \mathrm{~d}$. The results allow one to the find the three patterns correctly as can be seen in Figure 6e. 


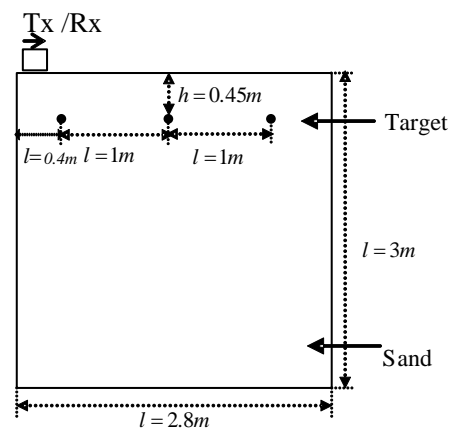

(a)

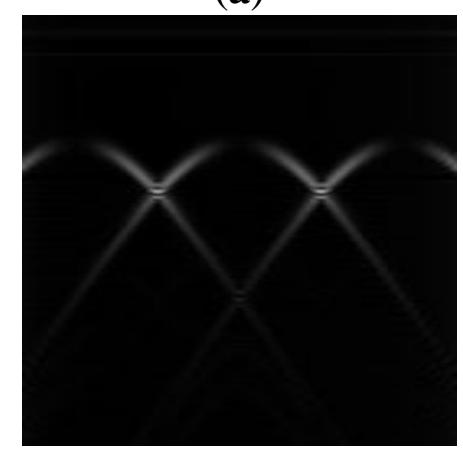

(c)

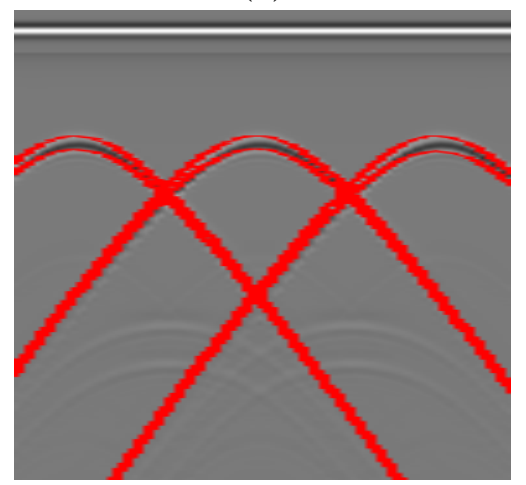

(e)

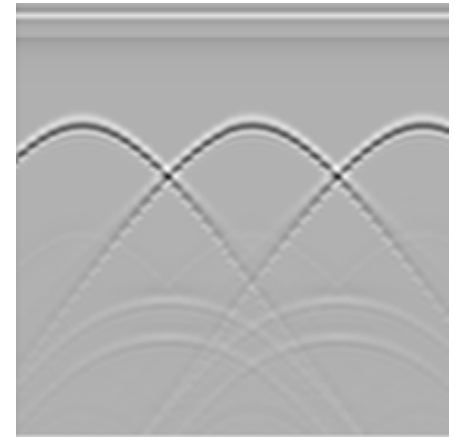

(b)

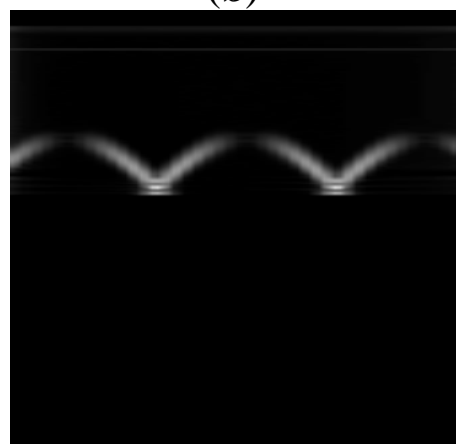

(d)

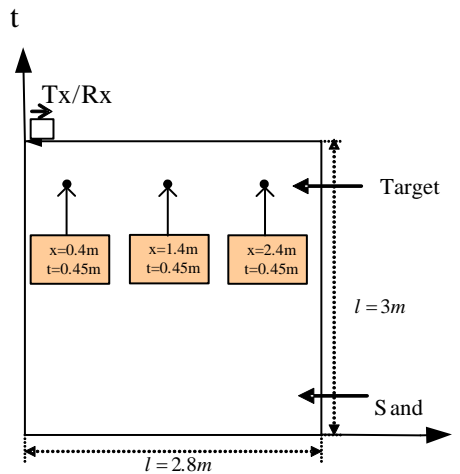

(f)

Figure 6. Example of MMSA detection result. (a) The location in reality; (b) GPR image; (c) Extraction of the amplitude component using MMSA; (d) Extraction the ROI of the amplitude component; (e) The hyperbola result of the Hough transform; (f) The position estimation result of the detection method.

Another result is presented in Figure 7. Figure 7 is an image simulated with the FDTD method. The width and depth are $2.8 \mathrm{~m}$ and $3 \mathrm{~m}$, respectively. There are three perfect metal point targets with the same $x$ coordinates. The depth of the top one is $0.2 \mathrm{~m}$ below the ground surface and the interval of the other two targets is $0.25 \mathrm{~m}$. The antenna receives a trace for each $2 \mathrm{~cm}$. There are 141 traces in total which contain 1060 samples. The interval between samples is $2.3587 \times 10^{-11} \mathrm{~s}$. The actual electromagnetic wave velocity is $14.5 \mathrm{~cm} / \mathrm{ns}$. Each detection result is processed by the Hough transform. This method detects the three patterns correctly. 


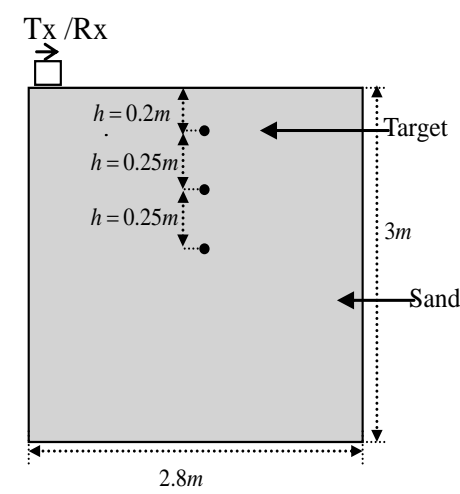

(a)

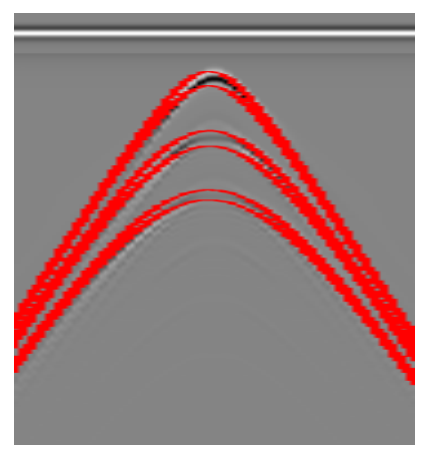

(c)

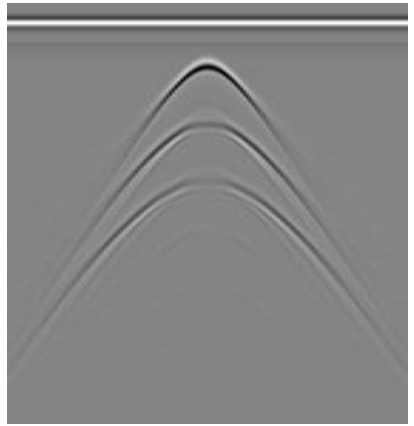

(b)

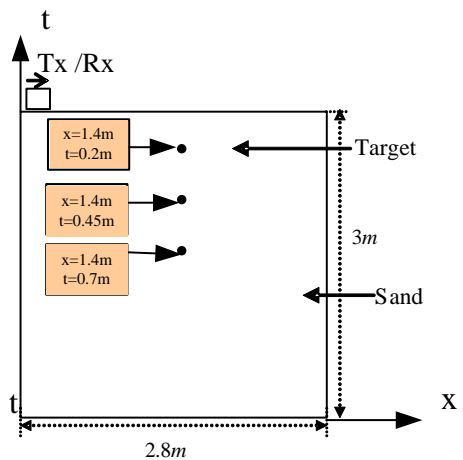

(d)

Figure 7. One more example of using MMSA. (a) Ground truth of the image; (b) GPR image; (c) The hyperbola result of the Hough transform; (d) The position estimation result of the detection method.

\subsection{Experiment Results}

Then we get GPR images in realistic situations. The first example is shown in Figure 8a, which is data from a real GPR experiment. Four metal spheres of $0.02 \mathrm{~m}$ diameter were buried under dry sand. These spheres were located side by side. The figure records the detection data with mean frequency of $200 \mathrm{MHz}$ and time window of $80 \mathrm{~ns}$. Finally, the detection result is attached to the picture (see Figure $8 \mathrm{~b}$ ). Further, $x_{0}$ and $v$ are estimated by fitting the extracted hyperbola. After the Hough transform, we get that the velocity of the data is $v=1.38 \times 10^{8} \mathrm{~m} / \mathrm{s}$, which is tested with the position of the targets in the real situation. As illustrated in Figure 8c, there are four targets in the ground. According to the extracted hyperbolic vertex coordinates, the antenna position of the target is shown in Figure 8c. For example, the vertex coordinate of the left target is $x_{1}=50$, so the antenna position of the target is $3 \mathrm{~m}$, which is the same as the position of the target in reality. Besides, the vertex coordinates axis of the hyperbola represents the depth of the target. The vertex coordinate of the left target is $t_{1}=97$.

A second example provided by the MMSA method is shown in Figure 9. Three similar landmines with a plastic case were buried under dry sand. The mines are Type-72A. The diameter of the landmine is $7.7 \mathrm{~cm}$ and the height is $3.7 \mathrm{~cm}$. Figure 9a shows the final detection result with the proposed MMSA method. Note that the proposed detection scheme provides a high detection rate even though the target is not so obvious. Figure $9 \mathrm{a}$ is a real GPR data profile. It recorded the point data with a mean frequency of $200 \mathrm{MHz}$ and time window of $50 \mathrm{~ns}$. The detection result is shown in Figure 9b, which illustrates that all patterns are identified with high precision.

The last example is shown in Figure 10, which illustrates the effects of the proposed MMSA detector and the final detection results. Note that the proposed detection scheme provides a high detection rate. Figure 10a is a real GPR data profile. It is recorded landmine detection data with a mean 
frequency of $200 \mathrm{MHz}$ and time window of $100 \mathrm{~ns}$. This is a two dimensional image. The detection result is illustrated in Figure 10b, which shows that all targets are localized.

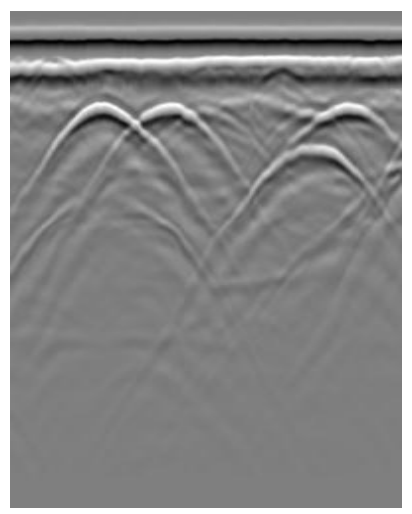

(a)

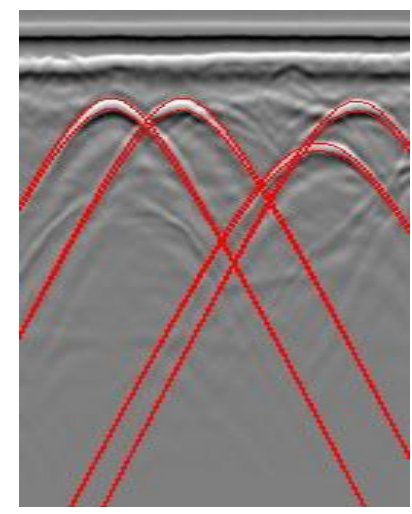

(b)

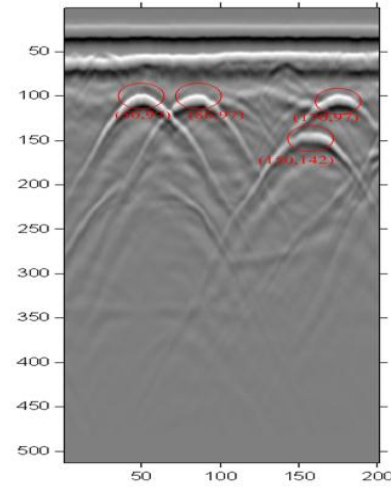

(c)

Figure 8. Example of test metal point targets data. (a) GPR image; (b) Processed result with detected patterns. The detected hyperbola is the red color line; (c) The position estimation results of the real point data.

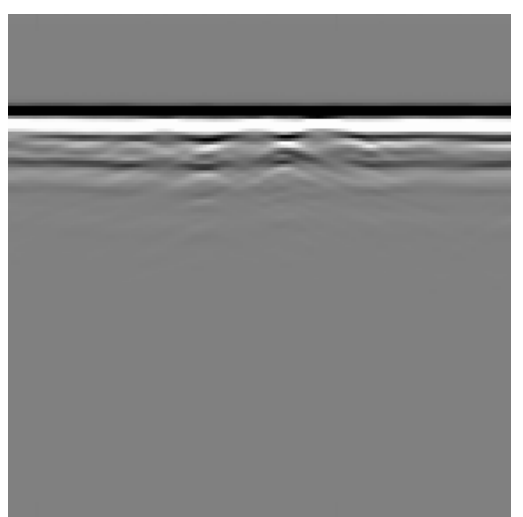

(a)

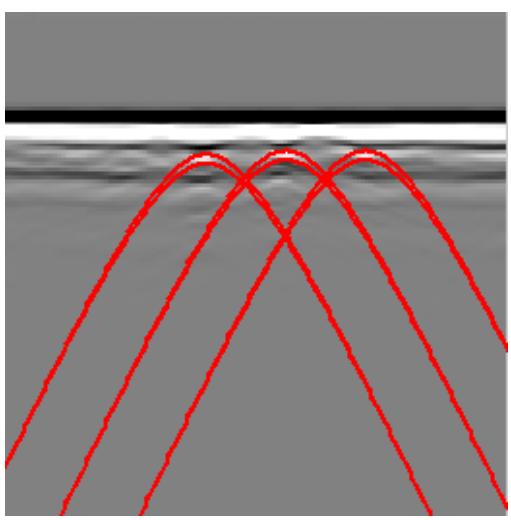

(b)

Figure 9. Example of landmine detection results. (a) GPR image; (b) Processed image with detected patterns.

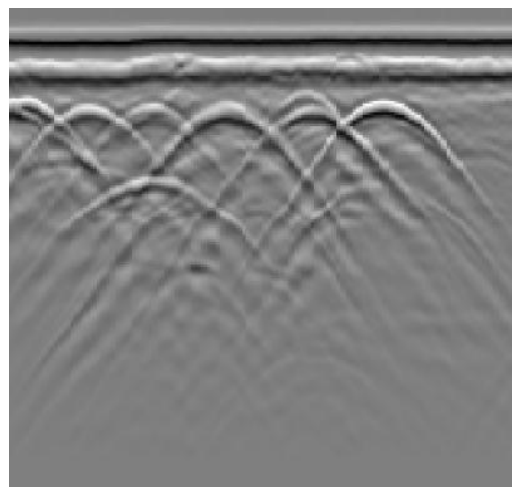

(a)

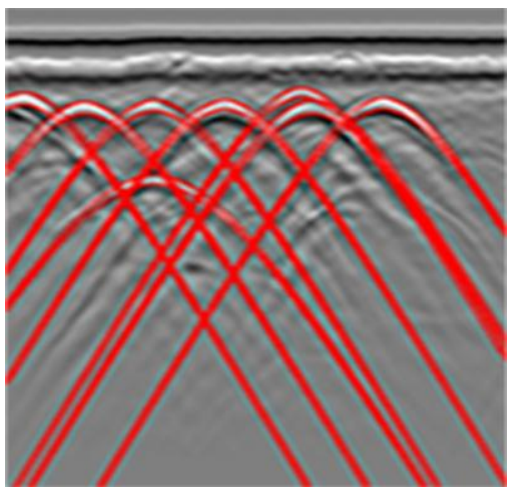

(b)

Figure 10. Example of test metal point targets data. (a) Real GPR image; (b) Processed results with detected patterns. 
We compare different aspects of the output of K-means+CEM [12], Hyperbolas Automatic Detection algorithm (HADA) [13] with the MMSA method:

1. How many hyperbolas are recognized correctly (true positives) and how many non-hyperbolas/ non-targets are recognized as hyperbolas (false positives)?

2. The distance error of all correctly recognized targets.

For the first aspect we investigate three different measurements:

$$
\begin{gathered}
\text { Recall }=\frac{\text { number of true positives }}{\text { number of all actual hyperbolas }} \\
\text { Precision }=\frac{\text { number of true positives }}{\text { num. of all hyperbolas (true }+ \text { false positives) }} \\
F-\text { measure }=2 \cdot \frac{\text { Precision } \cdot \text { Recall }}{\text { Precision }+ \text { Recal }}
\end{gathered}
$$

Table 1 show the different results of MMSA, HADA and K-means+CEM. The comparison in Table 1 shows that MMSA improves Precision and Recall as well as the position estimates. These improvements result from the fact that MMSA detects every hyperbola cluster. For K-means+CEM many position estimates have two errors larger than $10 \mathrm{~cm}$, resulting in bad values for "Recall" and "Precision". For correctly recognized targets there is still a distance error of a few centimeters. The reason is that $\mathrm{K}$-means+CEM method has problems with nearby and tangent hyperbolas as well as with background noise. For the HADA algorithm, the values for Recall and Precision are higher than with K-means+CEM, but there are still two position estimate errors larger than $10 \mathrm{~cm}$. MMSA achieves much better results than the other methods with the highest values of Recall and Precision.

Table 1. Results for data set 1 (Recall, Precision and F-measure with respect to hyperbolas).

\begin{tabular}{ccccc}
\hline Method & Recall (Hyperbolas) & Precision (Hyperbolas) & F-measure (Hyperbolas) & Distance Error(cm) \\
\hline K-means+CEM & 0.62 & 0.62 & 0.62 & 34.8 \\
HADA & 0.75 & 0.75 & 0.75 & 22.8 \\
MMSA & 1 & 1 & 0 & 5.8 \\
\hline
\end{tabular}

\section{Conclusions}

In this essay, target detection using MMSA has been demonstrated. This method detects buried objects and estimates their position correctly. The method combines the characteristics of wavelet and energy extraction. The amplitude component of the data is extracted using an unsupervised strategy based on MMSA. After the extraction of the amplitude component of the original date, background noise and direct wave are removed to large extent. We use the ROI extraction method to locate the genuine target area among spurious reflections. Then the labeled area is processed by the Hough transform to search the target's parameters. Finally, we estimate the horizontal and vertical position of the target. Experiments on many different GPR images show that the proposed method provides a general hyperbola-detection system with good results.

Acknowledgments: The authors thank K. Balac (KB) for her implementation of the Laplace/Riesz transform as a JAVA Plug in for Image and Kaifu Niu for his work on the language editing. This research is financially supported by the National Natural Science Foundations of China (under Grant Nos. 11201120, 61201389, 61201390); Key Scientific Research Project Program of Universities of Henan Province (under Grant No. 15A120001); Plan For Scientific Innovation Talent of Henan University of Technology(under Grant No. 2012CXRC07); Provincial Colleges and Universities on Basic Research Fund of Henan Technology University(under Grant No. 2014YWQQ11) and China Grain Public Service Research Projects (under Grant No. 201413003). Open fund of Key Laboratory of Grain Information Processing and Control (under Grant No. KFJJ-2015-102).

Author Contributions: Qiao, the principal author, contributed mostly to the literature survey and digestion in the classicaland new fields of information theory, radar and MMSA. She was also responsible for the research 
design, results analysis and interpretation and writing the paper. The primary contribution of Qin, the co-author, include the literature review, research-related experimentation and manuscript formatting.

Conflicts of Interest: The authors declare no conflict of interest.

\section{References}

1. Delbo, S.; Gamba, P.; Roccato, D. A fuzzy shell clustering approach to recognize hyperbolic signatures in subsurface radar images. IEEE Trans. Geosci. Remote Sens. 2000, 38, 1447-1451. [CrossRef]

2. Gamba, P.; Lossani, S. Neural detection of pipe signatures in ground penetrating radar images. IEEE Trans. Geosci. Remote Sens. 2000, 38, 790-797. [CrossRef]

3. Kaneko, T. Radar image processing for locating underground linear objects. IEICE Trans. 1991, 74, 3451-3458.

4. Shihab, S.; Al-Nuaimy, W.; Eriksen, A. Image processing and neural network techniques for automatic detection and interpretation of ground penetrating radar data. In Proceedings of the 6th International Multi-Conference on Circuits, Systems, Communications and Computers, Cancun, Mexico, 12-16 May 2002.

5. Capineri, L.; Grande, P.; Temple, J.A.G. Advanced image-processing technique for real-time interpretation of ground-penetrating radar images. Int. J. Imaging Syst. Technol. 1998, 9, 51-59. [CrossRef]

6. Unser, M.; Sage, D.; van de Ville, D. Multiresolution monogenic signal analysis using the Riesz-Laplace wavelet transform. IEEE Trans. Image Process. 2009, 18, 2402-2418. [CrossRef] [PubMed]

7. Dong, G.; Wang, N.; Kuang, G. Sparse representation of monogenic signal: With application to target recognition in SAR images. IEEE Signal Process. Lett. 2014, 21, 952-956.

8. Dong, G.; Kuang, G. Target recognition in SAR images via classification on Riemannian manifolds. IEEE Geosci. Remote Sens. Lett. 2015, 12, 199-203. [CrossRef]

9. Felsberg, M.; Sommer, G. The monogenic scale-space: A unifying approach to phase-based image processing in scale-space. J. Math. Imaging Vis. 2004, 21, 5-26. [CrossRef]

10. Chen, D.L. ROI Extraction Research of Ground Penetrating Radar Data. Bachelor Thesis, National University of Defense Technology, Changsha, China, 2003.

11. Huang, K.; Fu, K.; Sheen, T.; Cheng, S. Image processing of seismograms: Hough transformation for the detection of seismic patterns; thinning processing in the seismogram. Pattern Recog. 1985, 18, 429-440. [CrossRef]

12. Chen, H.; Cohn, A.G. Probabilistic robust hyperbola mixture model for interpreting ground penetrating radar data. In Proceedings of the 2010 International Joint Conference on Neural Networks, Barcelona, Spain, 18-23 July 2010.

13. Simi, A.; Bracciali, S.; Manacorda, G. Hough transform based automatic pipe detection for array GPR: Algorithm development and on-site tests. In Proceedings of the 2008 IEEE Radar Conference, Rome, Italy, 26-30 May 2008; pp. 1-6.

(C) 2015 by the authors; licensee MDPI, Basel, Switzerland. This article is an open access article distributed under the terms and conditions of the Creative Commons by Attribution (CC-BY) license (http://creativecommons.org/licenses/by/4.0/). 